\title{
Avant-propos
}

Mon intérêt pour l'histoire de l'anesthésie a commencé en 1993, au retour d'un voyage en Suède, où j'avais été invitée, en tant que Secrétaire de la Société d'odontologie pédiatrique de Strasbourg, à suivre les activités du professeur Anna-Lena Hallonsten, à l'Institute for Postgraduate Dental Education, à Jönköping. La prise en charge et les conditions dans lesquelles se déroulaient les soins des enfants handicapés, souvent déficients mentaux, m'avaient profondément impressionnée. Assis dans leurs chaises roulantes, les enfants repartaient heureux, après avoir été traités, sans la moindre appréhension, sous analgésie relative au protoxyde d'azote-oxygène. L'équipe médicale et dentaire de cette institution spécialisée était merveilleusement bien entraînée à ce type d'exercice. Les enfants "à problèmes » étaient soignés normalement, les dents cariées n'étaient pas extraites de manière systématique et les bouches ne présentaient pas d'édentations complètes. Il n'existait alors aucune structure similaire en France.

Comprendre ce qu'était cette forme d'anesthésie et pour quelles raisons elle avait été interdite, chez nous, au moment même où j'entrais dans la vie professionnelle (1971), fut le point de départ de ces recherches historiques. Ayant inventorié l'ensemble des brevets d'inventions du XIX ${ }^{\text {e }}$ siècle qui se rapportaient de près ou de loin à l'anesthésie et à la réanimation, l'idée m'était venue de les intégrer dans le contexte général du développement scientifique et médical, en cherchant, autant que possible, à établir mon argumentation d'après des documents et des manuscrits originaux. Seules les archives pouvaient me fournir des informations précises, des renseignements fiables, sur cette médecine du XIX siècle, dont de nombreux personnages et de nombreux récits ont été oubliés.

Ce livre est donc avant tout un ouvrage de référence, conçu à partir de textes autographes et de correspondances inédites. L'étude de l'histoire de l'anesthésie a été maintes fois abordée, dans des livres et des articles, par la compilation des sources imprimées. Celles-ci ne sont que les parties émergées d'un vaste corpus dont les fonds d'archives recèlent probablement encore des richesses insoupçonnées. Ce livre a donc pour but de faire revivre certains fonds et de permettre une nouvelle approche de l'histoire de cette spécialité. Il reste bien entendu à entreprendre des recherches plus approfondies dans d'autres centres d'archives, non visités vu l'ampleur de la tâche.

Il m’a semblé important de commencer ce travail au moment où la chimie fit un véritable bond en avant, au moment où la médecine tentait de sortir de l'empirisme, à l'instant où les nouvelles applications industrielles étaient mises à profit pour exploiter les minéraux et, finalement, où la thérapeutique médicale en fit un large usage. L'étude du développement de la chimie de quelques gaz propres à endormir la sensibilité, et celle de la médecine inhalatoire, l'histoire de la préparation de l'éther, de ses dérivés, du chloroforme, du protoxyde d'azote, celle des appareils utilisés pour administrer ces agents anesthésiques, entre la fin du $\mathrm{XVIII}^{\mathrm{e}}$ et la première moitié du XIX ${ }^{\mathrm{e}}$ siècle, permettent de comprendre comment l'anesthésie, cette 
révolution médicale, a réussi à pulvériser les anciennes habitudes chirurgicales et à supprimer l'effroyable peur du malade souffrant.

Un autre but de cet ouvrage, issu d'une thèse soutenue à l'École Pratique des Hautes Études, de la Sorbonne, à Paris, a été de tenter de répondre aux questions posées par les brevets d'invention délivrés par l'Institut National de la Propriété Industrielle. Étaient-ils uniquement le reflet de l'évolution des techniques industrielles ? Ont-ils trouvé une application réelle dans le domaine chirurgical, ou sont-ils tombés très rapidement dans l'oubli ? La conservation des appareils d'anesthésie permet certes d'enrichir nos connaissances historiques dans un domaine aussi pointu que l'anesthésiologie, mais encore fallait-il rendre compte des tâtonnements auxquels s'étaient livrés les fabricants d'instruments chirurgicaux avant d'aboutir à la conception d'un inhalateur de qualité. Seuls les manuscrits des brevets d'invention pouvaient nous en révéler les détails techniques, tant au niveau de l'encombrement de certains appareils que sur leur fonctionnement. Ils font ressurgir du passé les noms d'inventeurs, de scientifiques, de médecins et de pharmaciens dont on ne soupçonnait même pas l'existence.

J'ai délibérément omis de parler du brevet d'invention $n^{\circ} 4848$, déposé à l'United States Patent Office par Charles Thomas Jackson et William Green Morton, le 12 novembre 1846. Barbara Duncum en a reproduit sa spécification, en 1947, dans l'Appendix A de son livre The Development of Inhalation Anesthesia. Elle a également présenté le brevet (n $\left.{ }^{\circ} 5365\right)$ d'Augustus A. Gould et William Green Morton, qui avait été déposé au County of Suffolk and State of Massachusetts, le 13 novembre 1847.

J'ai éprouvé le besoin, dans une première partie de l'ouvrage, de clarifier les idées au sujet de la période pré-anesthésique, de la fin du XVIII e siècle à 1846. Mais il reste sans doute encore bien des choses à découvrir à ce sujet. La deuxième partie traite de la période empirique de l'anesthésie chirurgicale à l'éther sulfurique; la troisième, de l'anesthésie au chloroforme et de la diversité des nouveaux composés chimiques expérimentés par les physiologistes et les médecins pour soulager un malade ; la quatrième, de l'anesthésie au protoxyde d'azote et des nouveaux procédés dans l'art d'éthériser. Une cinquième partie examine la question de l'anesthésie générale au chlorure d'éthyle, ainsi que des inhalateurs utilisés au début du $\mathrm{xx}^{\mathrm{e}}$ siècle. Nous aborderons ensuite, dans une sixième partie, la préparation de l'oxygène et l'oxygénothérapie, et terminerons par quelques considérations sur le chloral et ses applications médico-chirurgicales.

Ce livre intéressera les historiens de l'anesthésie, ainsi que les historiens de la médecine, de la chimie, de l'industrie, et tous les lecteurs que l'histoire du XIX siècle touche autant que moi. 


\section{PREMIÈRE PARTIE}




\section{This page intentionally left blank}

